\title{
Thin Layer Chromatographic analysis for detecting various bioactive compounds in the ethanolic leaf extracts of Thevetia peruviana (Pers.) K. Schum [Thevetia White]
}

\author{
Krati Ghavri ${ }^{1}$, Madhavi Adhav ${ }^{2}$ \\ ${ }^{1,2}$ Department of Botany, P.M.B. Gujarati Science College, Indore, Madhya Pradesh, India
}

\begin{abstract}
The present study is aimed to investigate the bioactive compounds present in the tested plant by using Thin Layer Chromatography (TLC). Thevetia peruviana (Pers.) K. Schum is one of the important medicinal as well as ornamental plant belongs to the Dogbane family Apocynaceae. It is commonly known as yellow oleander. The plant is popular in traditional medicine especially for treatment of heart problems. The leaves are emetic and purgative. Leaf decoction is given to prevent conception. Thin layer chromatographic analysis reveals the presence of different bioactive compounds like alkaloids, flavanoids, cardiac glycosides, phenolic compounds, phytosterols and tannins, which are mainly contributed to medicinal utility of the plant, hence, Thevetia White ethanolic leaf extract can be used for drug preparation in pharmaceutical industries.
\end{abstract}

Keywords: Thin Layer Chromatography, cardiac glycosides, phytochemical analysis, Thevetia peruviana (Pers.) K. Schum.

\section{INTRODUCTION}

The plant kingdom contributed immensely to human health when no synthetic medicines were available, and when no concept of surgery existed. India is endowed with a rich wealth of medicinal plants. These plants have made a good contribution to the development of ancient Indian Materia Medica.

It is estimated that about 2, 50,000 to 5, 00,000 plant species present on the earth possess medicinal properties, which are used to cure a number of disorders and diseases. In most part of Asia, traditional plants have been part of their culture.( Prajapati et al.,2007; Kaushik and Dhiman ,1999).

Thevetia peruviana (Pers.) K. Schum is a small tree; $15-20 \mathrm{ft}$. high belongs to the Dogbane family Apocynaceae. It is a native of South America and West Indies. Leaves are simple, linear - lanceolate and whorled.(Fig No. 1).All parts of this plant abound in a milky juice which is highly poisonous.(Chopra et al.,1984).

The plant (Thevetia Yellow) is bitter, pungent, acrid, astringent to the bowels, useful in urethral discharges, worms, skin diseases, leucoderma, wound piles, eye trouble, itching ,fever and bronchitis.(Kirtikar and Basu,1981 ). The cardiac glycosides obtained from bark, kernals and flowers (Thevetia Yellow) are useful for heart diseases. (Prajapati $e t$ al.,2007) The root of this plant are made into a paste and applied to tumours. (Singh and Dey,2005).

The leaves are emetic and purgative. Leaf decoction is given to prevent conception. The purified glycosides thevetin extracted from the seed is prescribed as a cardio tonic drug. Seeds used as an abortifacient and purgative in rheumatism and dropsy; also used as an alexeteric. Diluted latex is given to treat irregular menstruation. (Ambasta,1986; Kaushik and Dhiman ,1999; Retnam and Martin,2006). 
Vol. 8, Issue 11, November 2021

DOI: 10.17148/IARJSET.2021.81107

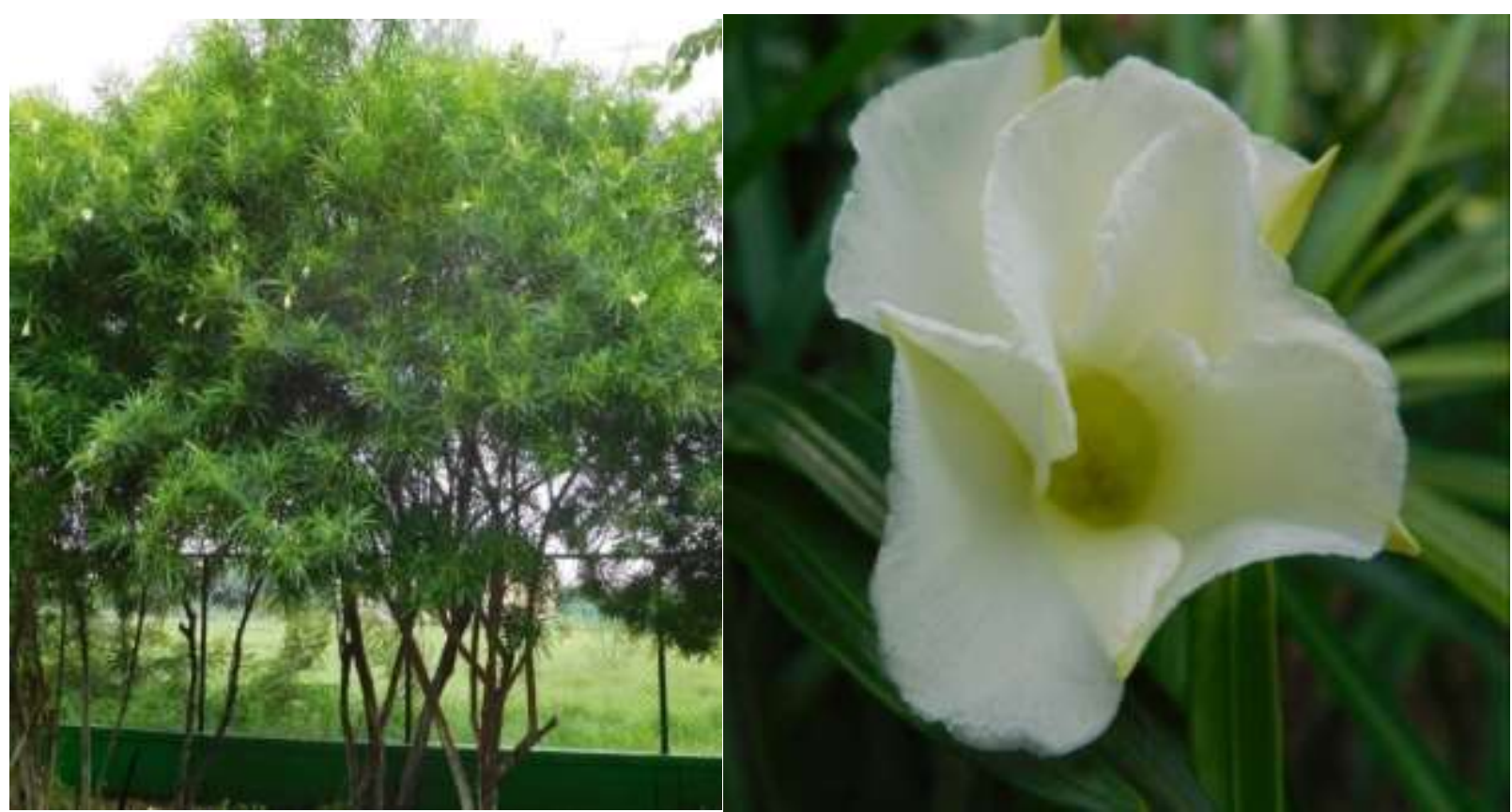

Fig 1: Thevetia peruviana (Pers.) K. Schum [Thevetia White]

\section{MATERIAL AND METHODS}

\section{Collection of Plant Material}

Plant materials (Thevetia White leaves) of Thevetia peruviana (Pers.) K. Schum were collected from Nehruvangram, Indore. The collected plant materials were identified with the help of Flora of Madhya Pradesh. (Mudgal et al.,1997).

\section{Extraction}

To obtain ethanolic extract $100 \mathrm{gm}$. of shade dried plant material was extracted with $500 \mathrm{ml}$. of ethanol (95\%) in "Soxhlet Extraction Apparatus. Finally the prepared plant material was macerated with water for 24 hrs. to obtain aqueous extract. Each extract was concentrated by distilling off the solvent (Kokate, 1994 and Kokate et al., 1993).

\section{Preliminary Phytochemical Screening}

The extract thus obtained was than subjected to preliminary phytochemical screening for identification of various plant constituents by methods suggested by (Finar, 1962; Farnsworth, 1966; Harborne, 1973; Harborne et al., 1979).

\section{Thin Layer Chromatography (TLC)}

Each ethanolic extract was than subjected to Thin Layer Chromatography by methods suggested by Kokate (1994) Stahl (1969), Wagner et al.(1984), Braithwaite and Smith (2009), Indhumathi and Mohandas (2013). The absorbent silica gel $\mathrm{GF}_{256}$ was coated to a thickness of $0.3 \mathrm{~mm}$ on clean TLC plates by commercial spreader.

The plates were activated at $105^{\circ} \mathrm{C}$ for 30 minutes and used. Rf values were calculated. Various solvent systems were used to detect the phytochemical constituents. The selection of mobile phase depends upon, type of constituents to be analyzed.. Here (8) different mobile phases were used.

\section{OBSERVATIONS AND DISCUSSION}

\section{Phytochemical screening}

The leaf extract of Thevetia White reveals the presence of alkaloids, flavanoids, glycosides-cardiac glycosides, phenolic compounds, tannins, phytosterols, carbohydrates, saponins, terpenoids, proteins and amino acids was noted in the observation Table, while fixed oils, fats, gums and mucilages were found absent.(Table No. 01).

Table No.1: Phytochemical screening of ethanolic leaf extract of Thevetia peruviana (Pers.) K. Schum [Thevetia White] 
International Advanced Research Journal in Science, Engineering and Technology

Vol. 8, Issue 11, November 2021

DOI: $10.17148 /$ IARJSET.2021.81107

\begin{tabular}{|c|c|c|}
\hline S. No. & Plant Constituents Test/Reagents & Results \\
\hline \multirow[t]{5}{*}{1.} & Alkaloids & \\
\hline & Mayer's reagent & + \\
\hline & Dragendorff's reagent & + \\
\hline & Hager's reagent & + \\
\hline & Wagner's reagent & + \\
\hline \multirow[t]{4}{*}{2.} & Carbohydrates & \\
\hline & Molish's reagent & + \\
\hline & Benedict's reagent & + \\
\hline & Fehling solution & + \\
\hline \multirow[t]{6}{*}{3.} & Types of Carbohydrates & \\
\hline & Glucose & + \\
\hline & Fructose & + \\
\hline & Galactose & - \\
\hline & Lactose & + \\
\hline & Starch & + \\
\hline \multirow[t]{2}{*}{4.} & Phytosterols & \\
\hline & Liebermann-Burchard's test & + \\
\hline \multirow[t]{2}{*}{5.} & Terpenoids & \\
\hline & Salkowski reaction & + \\
\hline \multirow[t]{2}{*}{6.} & Fixed oils and fats & \\
\hline & Spot test & - \\
\hline \multirow[t]{2}{*}{7.} & Saponins & \\
\hline & Foam test & + \\
\hline \multirow[t]{2}{*}{8.} & Phenolic compounds & \\
\hline & Ferric chloride solution & + \\
\hline \multirow[t]{2}{*}{9.} & Tannins & \\
\hline & Lead acetate solution & + \\
\hline \multirow[t]{3}{*}{10.} & Proteins & \\
\hline & Biuret test & + \\
\hline & Xanthoprotic test & + \\
\hline \multirow[t]{2}{*}{11.} & Amino acids & \\
\hline & Ninhydrin reagent & + \\
\hline \multirow[t]{2}{*}{12.} & Gums and mucilages & \\
\hline & Alcoholic precipitation & - \\
\hline \multirow[t]{3}{*}{13.} & Flavanoids & \\
\hline & Shinoda test & + \\
\hline & Lead acetate test & + \\
\hline \multirow[t]{2}{*}{14.} & Cardiac glycosides & \\
\hline & Killer kiliani test & + \\
\hline
\end{tabular}

+Present - Absent

Thin Layer Chromatography (TLC)

In leaf extracts maximum separation was found in Chloroform: Methanol: Water (7:4:1) mobile phase which is used for the detection of phytosterols. The result of TLC analysis reveals the presence of alkaloids, amino acids, essential oils, flavanoids, glycosides, phenolic compounds, phytosterols, saponins, tannins and terpenoids. 
International Advanced Research Journal in Science, Engineering and Technology

Vol. 8, Issue 11, November 2021

DOI: $10.17148 /$ IARJSET.2021.81107

Fig.No.2: TLC observations of ethanolic leaf extracts of Thevetia White in different mobile phase. Alkaloids Amino acids Essential oils

Flavanoids

Glycosides
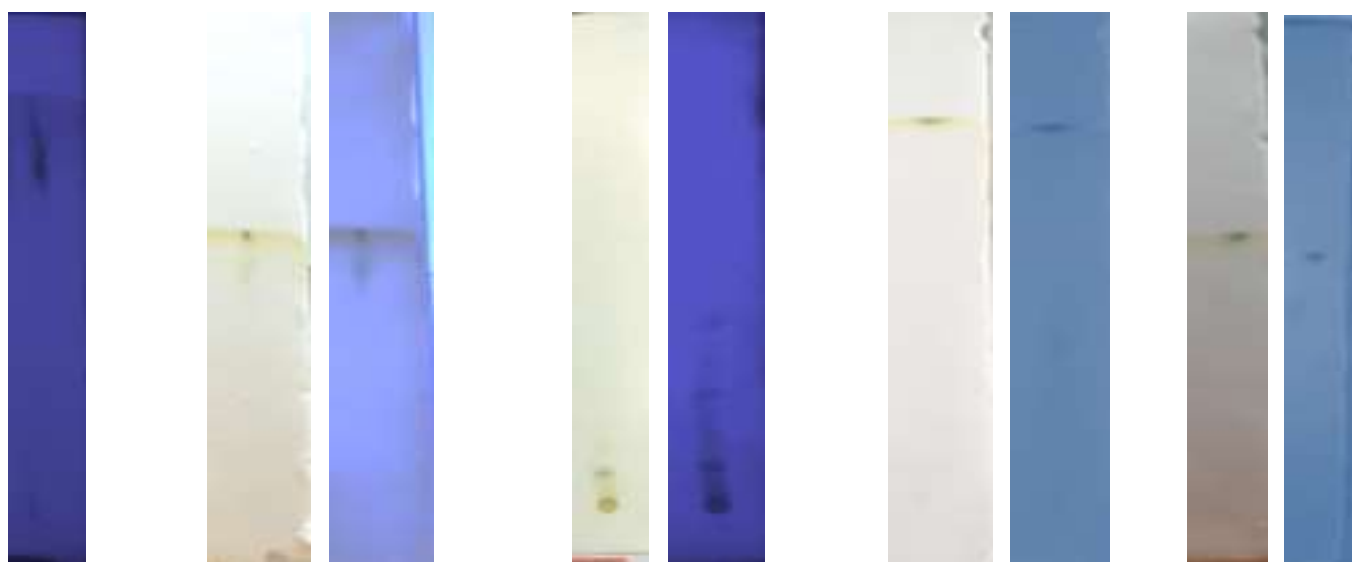

Visible light UV lamp Visible light UV lamp Visible light UV lamp Visible light UV lamp Visible light UV lamp
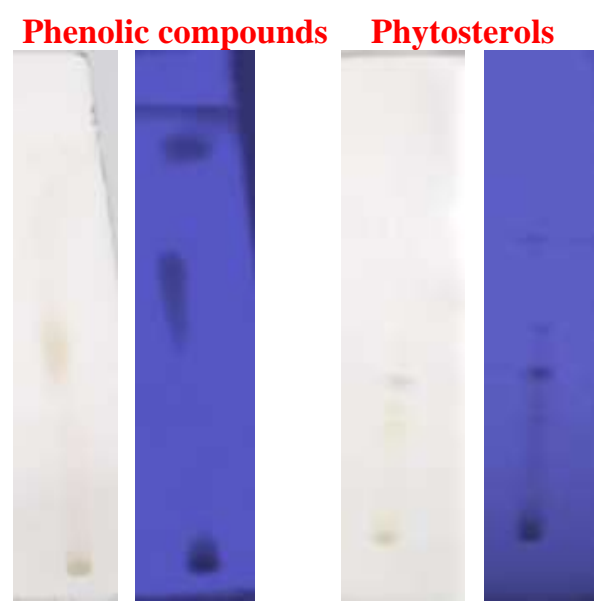

Saponins

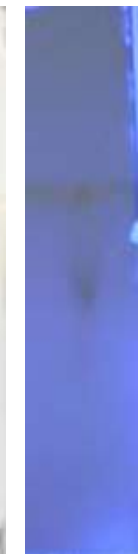

Visible light UV lamp Visible light UV lamp Visible light Visible light UV lamp

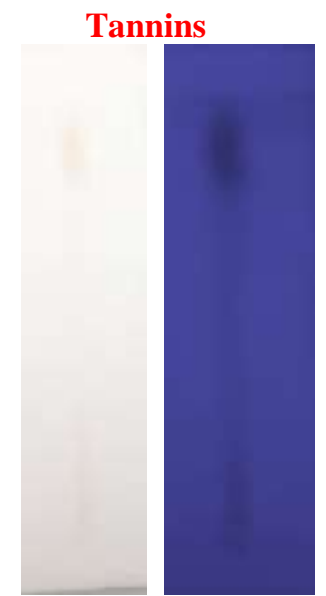

UV lamp Visible light
Terpenoids

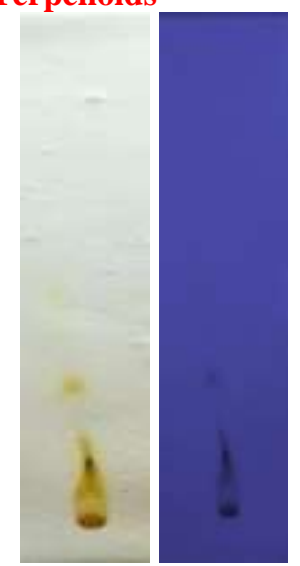

UV lamp

Table No. 2 : TLC observations of different phytoconstituents from ethanolic leaf extracts of Thevetia peruviana (Pers.) K. Schum. [Thevetia White]

\begin{tabular}{|c|c|c|c|c|c|c|c|c|}
\hline \multirow{2}{*}{$\begin{array}{l}\text { S. } \\
\mathbf{N} \\
\text { o. }\end{array}$} & \multirow{2}{*}{$\begin{array}{l}\text { Name of the } \\
\text { Phytoconstit } \\
\text { uents }\end{array}$} & \multirow[t]{2}{*}{ Mobile phases } & \multicolumn{3}{|l|}{ Eyes } & \multicolumn{3}{|c|}{ UV light } \\
\hline & & & $\begin{array}{l}\text { Num } \\
\text { ber of } \\
\text { spots } \\
\text { on } \\
\text { TLC } \\
\text { plates }\end{array}$ & $\begin{array}{l}\text { Colou } \\
\mathbf{r}\end{array}$ & $\mathbf{R f}$ & $\begin{array}{l}\text { Num } \\
\text { ber of } \\
\text { spot } \\
\text { on } \\
\text { TLC } \\
\text { plates }\end{array}$ & Colour & $\mathbf{R f}$ \\
\hline 1. & Alkaloids & $\begin{array}{l}\mathrm{CHCL}_{3}: \text { Methanol : } \\
\text { Glacial acetic acid } \\
(83: 17: 10)\end{array}$ & 1 & Brown & 0.88 & 1 & $\begin{array}{l}\text { Greenish } \\
\text { blue }\end{array}$ & 0.88 \\
\hline 2. & Amino acids & $\begin{array}{l}\text { n-Butanol :acetic acid } \\
\text { :water }(4: 5: 1)\end{array}$ & $\begin{array}{l}1 \\
2\end{array}$ & $\begin{array}{l}\text { Brown } \\
\text { Green }\end{array}$ & $\begin{array}{l}0.85 \\
0.98\end{array}$ & $\begin{array}{l}1 \\
2\end{array}$ & $\begin{array}{l}\text { Brown } \\
\text { Greenish } \\
\text { brown }\end{array}$ & $\begin{array}{l}0.85 \\
0.98\end{array}$ \\
\hline
\end{tabular}


Vol. 8, Issue 11, November 2021

DOI: $10.17148 /$ IARJSET.2021.81107

\begin{tabular}{|c|c|c|c|c|c|c|c|c|}
\hline 3. & Essential oils & $\begin{array}{l}\text { Hexane : Acetone } \\
(9: 1)\end{array}$ & $\begin{array}{l}1 \\
2\end{array}$ & $\begin{array}{l}\text { Black } \\
\text { Yellow }\end{array}$ & $\begin{array}{l}0.18 \\
0.26\end{array}$ & $\begin{array}{l}1 \\
2 \\
3\end{array}$ & $\begin{array}{l}\text { Violet } \\
\text { Violet } \\
\text { Violet }\end{array}$ & $\begin{array}{l}0.18 \\
0.26 \\
0.40\end{array}$ \\
\hline 4. & Flavanoids & $\begin{array}{l}\text { Ethyl acetate :Methyl } \\
\text { ethyl ketone :Acetic } \\
\text { acid : Water(5:3:1:1) }\end{array}$ & 1 & Green & 0.97 & 1 & $\begin{array}{l}\text { Greenish } \\
\text { brown }\end{array}$ & 0.97 \\
\hline 5. & Glycosides & $\begin{array}{l}\text { Ethyl Acetate : } \\
\text { Benzene }(2: 1)\end{array}$ & 1 & Green & 0.94 & 1 & Brown & 0.94 \\
\hline 6. & $\begin{array}{l}\text { Phenolic } \\
\text { Compounds }\end{array}$ & $\begin{array}{l}n-\text { Butanol : Acetic } \\
\text { acid : Water (35: } 5 \text { : } \\
\text { 12) }\end{array}$ & 1 & Brown & 0.67 & $\begin{array}{l}1 \\
2\end{array}$ & $\begin{array}{l}\text { Violet } \\
\text { Violet }\end{array}$ & $\begin{array}{l}0.67 \\
0.92\end{array}$ \\
\hline 7. & Phytosterols & $\begin{array}{l}\text { P. ether : Ethyl } \\
\text { acetate }(7: 3)\end{array}$ & $\begin{array}{l}1 \\
2\end{array}$ & $\begin{array}{l}\text { Orange } \\
\text { Black }\end{array}$ & $\begin{array}{l}0.59 \\
0.72\end{array}$ & $\begin{array}{l}1 \\
2 \\
3\end{array}$ & $\begin{array}{l}\text { Green } \\
\text { Green } \\
\text { Violet }\end{array}$ & $\begin{array}{l}0.59 \\
0.72 \\
0.98\end{array}$ \\
\hline 8. & Saponins & $\begin{array}{l}\text { Chloroform } \\
\text { Methanol : } \\
(7: 4: 1)\end{array}$ & 1 & Orange & 0.95 & $\begin{array}{l}1 \\
2\end{array}$ & $\begin{array}{l}\text { Black } \\
\text { Brown }\end{array}$ & $\begin{array}{l}0.71 \\
0.95\end{array}$ \\
\hline 9. & Tannins & $\begin{array}{lr}\text { Chloroform: } & \text { Ethyl } \\
\text { acetate: } & \text { Ethanol } \\
(6: 4: 4) & \end{array}$ & 1 & Brown & 0.90 & 1 & Brown & 0.90 \\
\hline $\begin{array}{l}1 \\
\mathbf{0} .\end{array}$ & Terpenoids & $\begin{array}{l}\text { Ethyl acetate: } \\
\text { Chloroform (6:4) }\end{array}$ & $\begin{array}{l}1 \\
2\end{array}$ & $\begin{array}{l}\text { Brown } \\
\text { Brown }\end{array}$ & $\begin{array}{l}0.22 \\
0.36\end{array}$ & $\begin{array}{l}1 \\
2\end{array}$ & $\begin{array}{l}\text { Brown } \\
\text { Brown }\end{array}$ & $\begin{array}{l}0.22 \\
0.36\end{array}$ \\
\hline
\end{tabular}

\section{CONCLUSION}

This investigation has shown the preliminary informations to determine the chemical composition of the studied Thevetia peruviana (Pers.) K. Schum. plant . [Thevetia White] .Thin layer chromatographic analysis reveals the presence of different bioactive compounds like alkaloids, flavanoids, cardiac glycosides, phenolic compounds, phytosterols and tannins, which are mainly contributed to medicinal utility of the plant hence, Thevetia White ethanolic leaf extract can be used for drug preparation in pharmaceutical industries.

\section{ACKNOWLEDGEMENT}

We wish to express our sincere gratitude to the Principal and Head of the Department of Botany, P. M .B. Gujarati Science College, Indore, for provided full research laboratory facilities throughout our work.

\section{REFERENCES}

1. Ambasta, S.P. (1986).The useful plants of India. Publications and Information Directorate, CSIR, New Delhi, India.636

2. Braithwaite. A. and Smith F.J.(2009).Chromatographic methods. Kluwer academic publishers, Dord, 59-63.

3. Chopra , S.R.N.; Badhwar, R. L. and Ghosh ,S. (1984).Poisonous Plants of India. Academic Publishers, Jaipur, India .1:665-668.

4. Farnsworth, N. (1996).Biological and Phytochemical Screening of Plants. J. pharm. Sci. 55:225-276.

5. Finar ,L .L. (1962). Organic chemistry, Lonngman, Green Grosvent Street, London.

6. Harborne, J.B. (1973). Phytochemical methods, Chapman and Hall Ltd London.49-188.

7. Harborne,J.B.; Mabry,T.J.; Mabry, H. (1979).The flavonoids .Chapman and Hall International Edition, London.

8. Indumathi, T. and Mohandas, S. (2013) .Identification of bioactive components in Solanum incanum fruit by Thin Layer Chromatography and HPTLC. International journal of scientific research; 2(6):22-25.

9. Kaushik, P. and Dhiman, A.K.(1999).Medicinal plants and raw drugs of India. Bishen Singh Mahendra pal Singh publication, Dehradun, India. 352-353.

10. Kirtikar,K.R.and Basu,B.D.(1981).Indian medicinal plants. International book distributors.2: 1553-1556.

11. Kokate, C.K;Purohit.A.P.;Gokhale,B.B.(1993). Pharmacognosy, Twelth Edition, Nirali prakashan, Pune,India.90-93.

12. Kokate,C.K. (1994).Practical pharmacognosy ,Fourth Edition, Vallabh prakashan, Delhi,India.107-111.

13. Malviya, S. and Dwivedi,P.(2019). Extracts of Ailanthus excels an Essential Medicine in Ayurveda: Pharmacological evaluation and preliminary screening of phytochemicals. Journal of Drug Delivery and Therapeutics. 9(1):84-87. 
International Advanced Research Journal in Science, Engineering and Technology

Vol. 8, Issue 11, November 2021

\section{DOI: 10.17148/IARJSET.2021.81107}

14. Mudgal,V.;Khanna,K.K.;Hajra,P.K.(1997) Flora of Madhya Pradesh. The director, Botanical Survey of India, Calcutta.59-60.

15. Prajapati,N.D.;Purohit,S.S.;Sharma,A.K.;Kumar,T.(2007).A Handbook of medicinal plants.Agrobios,Jodhpur,India.511-512.

16. Rahman, N.; Rahman, H.; Haris, M.; Mahmood, R. (2019). Antioxidant, anti-inflammatory and wound healing properties of ethanolic extracts of Thevetia peruviana (Pers.) K. Schum. J. Res. Pharm; 23(1):101-113.

17. Retnam; Raveendra, K.;Martin ,P. (2006) Ethnomedicinal plants. Agrobios publication, India.

18. Singh M.P. and Dey S.(2005). Indian medicinal plants. Satish Serial Publishing House, Delhi.399.

19. Silpa M.; Joghee, S. and Hamsalakshmi (2020). A phytochemical study on Eupatorium glandulosum. Asian J Pharm Clin Res, 13 (1):77-80.

20. Stahl, E. (1969). Thin layer chromatography- A laboratory hand book. Springer Verlag, Berlin.

21. Wagner,H; Baldt, S and Zgainski, E.M. (1984).Plant drug analysis. A thin layer chromatography atlas. Springer-verlag, Berlin Heidelberg, New York Tokyo.1-309. 